\title{
ON ASYMPTOTIC EXPANSIONS FOR THE FRACTIONAL INFINITY LAPLACIAN
}

\author{
FÉLIX DEL TESO, JøRGEN ENDAL, AND MARTA LEWICKA
}

\begin{abstract}
We propose two asymptotic expansions of two interrelated integral-type averages, in the context of the fractional $\infty$-Laplacian $\Delta_{\infty}^{s}$ for $s \in\left(\frac{1}{2}, 1\right)$. This operator has been introduced and first studied in [Bjorland, C., Caffarelli, L. and Figalli, A., Nonlocal Tugof-War and the inifnity fractional Laplacian, Comm. Pure Appl. Math., 65, pp. 337-380, (2012)]. Our expansions are parametrised by the radius of the removed singularity $\varepsilon$, and allow for the identification of $\Delta_{\infty}^{s} \phi(x)$ as the $\varepsilon^{2 s}$-order coefficient of the deviation of the $\varepsilon$-average from the value $\phi(x)$, in the limit $\varepsilon \rightarrow 0+$. The averages are well posed for functions $\phi$ that are only Borel regular and bounded.
\end{abstract}

\section{INTRODUCTION}

This paper concerns the fractional $\infty$-Laplace operator $\Delta_{\infty}^{s}$, as introduced in [1] and represented by (1.8) below. Given a function $\phi: \mathbb{R}^{N} \rightarrow \mathbb{R}$, our main result is the identification of $\Delta_{\infty}^{s} \phi(x)$ as the $\varepsilon^{2 s}$-order coefficient in the asymptotic expansion of the deviation of an appropriate $\varepsilon$-average $\mathcal{A}_{\varepsilon}$ applied on $\phi$, from the value $\phi(x)$. Such identification is of general interest in the analysis of partial differential operators, their related probabilistic interpretation via Tug-of-War games, a study of viscosity solutions and of numerical approximating schemes. The chief example of the said asymptotic expansions is given by the well known (local and linear) formula for the Laplace operator, where $\Delta \phi(x)$ emerges as the $\varepsilon^{2}$-order coefficient from the integral average $f_{B_{\varepsilon}}$ :

$$
f_{B_{\varepsilon}(x)} \phi(y) \mathrm{d} y=\phi(x)+\frac{\varepsilon^{2}}{2(N+2)} \Delta \phi(x)+o\left(\varepsilon^{2}\right) \quad \text { as } \varepsilon \rightarrow 0+.
$$

The parallel expansion of the $\infty$-Laplacian: $\Delta_{\infty} \phi(x)=\left\langle\nabla^{2} \phi(x): \frac{\nabla \phi(x)}{|\nabla \phi(x)|} \otimes \frac{\nabla \phi(x)}{|\nabla \phi(x)|}\right\rangle$ utilizes the midpoint (local and nonlinear) average $\frac{1}{2}\left(\sup _{B_{\varepsilon}}+\inf _{B_{\varepsilon}}\right)$ in:

$$
\frac{1}{2}\left(\sup _{B_{\varepsilon}(x)} \phi+\inf _{B_{\varepsilon}(x)} \phi\right)=\phi(x)+\frac{\varepsilon^{2}}{2} \Delta_{\infty} \phi(x)+o\left(\varepsilon^{2}\right) \quad \text { as } \varepsilon \rightarrow 0+.
$$

2010 Mathematics Subject Classification. 35B05, 35J60, 35D40, 47G20.

Key words and phrases. Mean value formulas, nonlocal gradient dependent operators, fractional infinity Laplacian.

F. del Teso was partially supported by PGC2018-094522-B-I00 from the MICINN of the Spanish Government. J. Endal received funding from the Research Council of Norway under the Toppforsk (research excellence) grant agreement no. 250070 "Waves and Nonlinear Phenomena (WaNP)", from the European Union's Horizon 2020 research and innovation programme under the Marie Skłodowska-Curie grant agreement no. 839749 "Novel techniques for quantitative behavior of convection-diffusion equations (techFRONT)", and from the Research Council of Norway under the MSCA-TOPP-UT grant agreement no. 312021.

M. Lewicka was supported by NSF grants DMS-1613153 and DMS-2006439. 
1.1. The asymptotic expansions and averaging operators in this paper. In what follows, will prove that for every $s \in\left(\frac{1}{2}, 1\right)$ one counterpart formula of (1.1) for $\Delta_{\infty}^{s}$ is:

$$
\mathcal{A}_{\varepsilon}^{o} \phi(x)=\phi(x)+s \varepsilon^{2 s} \Delta_{\infty}^{s} \phi(x)+o\left(\varepsilon^{2 s}\right) \quad \text { as } \varepsilon \rightarrow 0+,
$$

based on the following (nonlocal and nonlinear) average:

$$
\mathcal{A}_{\varepsilon}^{o} \phi(x)=\frac{1}{2}\left(\sup _{|y|=1} f_{\varepsilon}^{\infty} \phi(x+t y) \mathrm{d} \mu_{s}(t)+\inf _{|y|=1} f_{\varepsilon}^{\infty} \phi(x+t y) \mathrm{d} \mu_{s}(t)\right) .
$$

The one-dimensional fractional measure $\mu_{s}$ and the structure of the error term $o\left(\varepsilon^{2 s}\right)$ will be explained below. When $\nabla \phi(x) \neq 0$, we also derive another identification through a localnonlocal average, which is a convex combination of the averages used in (1.3) and (1.2):

$$
\mathcal{A}_{\varepsilon} \phi(x)=(1-s) \cdot \mathcal{A}_{\varepsilon}^{o} \phi(x)+s \cdot \frac{1}{2}\left(\sup _{B_{\varepsilon}(x)} \phi+\inf _{B_{\varepsilon}(x)} \phi\right) .
$$

We anticipate that the error quantity $o\left(\varepsilon^{2 s}\right)$ below is uniform in the whole considered range $s \in\left(\frac{1}{2}, 1\right)$, whereas the corresponding error in (1.3) blows up to $\infty$ as $s \rightarrow 1-$. Thus, the following asymptotic expansion can be seen as an improvement of (1.3):

$$
\mathcal{A}_{\varepsilon} \phi(x)=\phi(x)+(1-s) s \varepsilon^{2 s} \Delta_{\infty}^{s} \phi(x)+o\left(\varepsilon^{2 s}\right) \quad \text { as } \varepsilon \rightarrow 0+.
$$

Precise statements of (1.3) and (1.6) will be given in Theorems 1.1, 1.2 and Remarks 3.3, 4.2

1.2. The fractional $\infty$-Laplacian. Let $\phi: \mathbb{R}^{N} \rightarrow \mathbb{R}$ be a bounded Borel function. We recall that $\phi \in C^{1,1}(x)$ at $x \in \mathbb{R}^{N}$, provided that there exists $p_{x} \in \mathbb{R}^{N}$ and $C_{x}, \eta_{x}>0$ such that:

$$
\left|\phi(x+y)-\phi(x)-\left\langle p_{x}, y\right\rangle\right| \leq C_{x}|y|^{2} \quad \text { for all }|y|<\eta_{x} \text {. }
$$

In [1, Definition 1.1], the (normalized) fractional $\infty$-Laplacian $\Delta_{\infty}^{s} \phi(x)$, for $s \in\left(\frac{1}{2}, 1\right)$, has been introduced by means of two distinct formulas, distinguishing between cases $p_{x}=0$ and $p_{x} \neq 0$. In section 2, we provide a rigorous proof of the following alternative definition stated in [1]:

$$
\Delta_{\infty}^{s} \phi(x)=\frac{1}{\alpha_{s}} \cdot \sup _{|y|=1} \inf _{|\tilde{y}|=1} \int_{0}^{\infty} L_{\phi}(x, t y, t \tilde{y}) \mathrm{d} \mu_{s}(t) .
$$

To explain the notation in the right hand side above, for each $x, y, \tilde{y} \in \mathbb{R}^{N}$ we define:

$$
L_{\phi}(x, y, \tilde{y}) \doteq \phi(x+y)+\phi(x-\tilde{y})-2 \phi(x) .
$$

Further, $\mu_{s}$ is the measure 1 on the Borel subsets of $(0, \infty)$, given by:

$$
\mathrm{d} \mu_{s}(t) \doteq \frac{\alpha_{s}}{t^{1+2 s}} \mathrm{~d} t \quad \text { where } \alpha_{s}=\frac{4^{s} s \Gamma\left(\frac{1}{2}+s\right)}{\pi^{1 / 2} \Gamma(1-s)}=\left(2 \int_{0}^{\infty} \frac{1-\cos t}{t^{1+2 s}} \mathrm{~d} t\right)^{-1} .
$$

It is important to note [10] that one can express $\alpha_{s}$ by means of another constant $c_{s}$, that is bounded and positive, uniformly in $s$. Namely, there holds:

$$
\alpha_{s}=s(1-s) c_{s} \text {. }
$$

We also point out that the operator $\Delta_{\infty}^{s}$ treated in this paper, is not the only nonlocal counterpart of $\Delta_{\infty}$. A variational (i.e. energy based) fractional infinity Laplacian was studied in [5] and a non normalized one in [6]. These operators are not suited for a game theoretical approach, which was the main motivation in [1].

\footnotetext{
${ }^{1}$ The role of the normalizing constant $\alpha_{s}$ is to ensure that the operator $-(-\Delta)^{s} u(x) \doteq \int_{0}^{\infty} L_{u}(x, t, t) \mathrm{d} \mu_{s}(t)$ defined for $u: \mathbb{R} \rightarrow \mathbb{R}$, is a pseudo-differential operator with symbol $|\xi|^{2 s}$.

${ }^{2} \mathrm{~A}$ direct calculation shows that, for example, $c_{s} \in\left(\left(\frac{12}{13}\right)^{2},\left(\frac{12}{5}\right)^{2}\right)$ in the range $s \in\left(\frac{1}{2}, 1\right)$.
} 
1.3. Statements and discussion of main results. We consider the operator in the right hand side of (1.8):

$$
\mathcal{L}_{s}[\phi](x) \doteq \sup _{|y|=1} \inf _{\tilde{y} \mid=1} \int_{0}^{\infty} L_{\phi}(x, t y, t \tilde{y}) \mathrm{d} \mu_{s}(t) .
$$

Given $\eta_{x}>0$, we work with the following hypotheses on $\phi$, relative to the ball $B_{\eta_{x}} \doteq B_{\eta_{x}}(x)$ :

(i) $\phi \in C^{2}\left(\bar{B}_{\eta_{x}}\right)$ where $p_{x} \doteq \nabla \phi(x)$ and $C_{x} \doteq \frac{1}{2}\left\|\nabla^{2} \phi\right\|_{L^{\infty}\left(B_{\eta_{x}}\right)}$.

(ii) $\phi$ is bounded and uniformly continuous on $\mathbb{R}^{N} \backslash \bar{B}_{\eta_{x}}$ with modulus of conti-

$$
\text { nuity } \left.\omega_{\phi}(a) \doteq \sup \left\{\left|\phi\left(y_{1}\right)-\phi\left(y_{2}\right)\right| ; y_{1}, y_{2} \in \mathbb{R}^{N} \backslash \bar{B}_{\eta_{x}},\left|y_{1}-y_{2}\right| \leq a\right\} . \quad\right\}
$$

Regularity required in $(\overline{\mathbf{H}})$ is satisfied by the test functions in the viscosity solution setting (see section 5.2). We further denote:

$$
\begin{aligned}
& A_{\varepsilon}=\max \left\{\frac{16 C_{x}}{\left|p_{x}\right|} \cdot \frac{2 s-1}{1-s} \cdot \frac{\eta_{x}^{2-2 s}-\varepsilon^{2-2 s}}{\varepsilon^{1-2 s}-\eta_{x}{ }^{1-2 s}}, \kappa_{\varepsilon}\right\}, \\
& \kappa_{\varepsilon}=\sup \left\{a ; a \in[0,2] \text { and } a^{2} \leq \frac{8 \omega_{\phi}(a)}{\left|p_{x}\right|} \cdot \frac{\frac{2 s-1}{2 s} \eta_{x}^{-2 s}+\eta_{x}^{1-2 s}}{\varepsilon^{1-2 s}-\eta_{x}^{1-2 s}}\right\} .
\end{aligned}
$$

Our first main result regards the expansion (1.3):

Theorem 1.1. Let $\phi: \mathbb{R}^{N} \rightarrow \mathbb{R}$ satisfy $(\underline{\mathbf{H}})$. Then there holds, for every $\varepsilon<\eta_{x}$ :

$$
\begin{aligned}
& \left|\mathcal{A}_{\varepsilon}^{o} \phi(x)-\phi(x)-\frac{1}{c_{s}(1-s)} \varepsilon^{2 s} \mathcal{L}_{s}[\phi](x)\right| \leq \frac{s}{1-s} \cdot C_{x} \varepsilon^{2}+ \\
& \quad+ \begin{cases}\varepsilon^{2 s}\left(4 s C_{x} \frac{\eta_{x}^{2-2 s}-\varepsilon^{2-2 s}}{1-s} \cdot A_{\varepsilon}+\left(\eta_{x}^{-2 s}+\frac{2 s}{2 s-1} \eta_{x}^{1-2 s}\right) \cdot \omega_{\phi}\left(A_{\varepsilon}\right)\right) & \text { when } p_{x} \neq 0 \\
0 & \text { when } p_{x}=0 .\end{cases}
\end{aligned}
$$

Our second main result regards the expansion (1.6):

Theorem 1.2. Assume ([-

$$
\begin{aligned}
\mid \mathcal{A}_{\varepsilon} \phi(x)- & \phi(x)-\frac{1}{c_{s}} \varepsilon^{2 s} \mathcal{L}_{s}[\phi](x) \mid \\
\leq & 2 \varepsilon^{2 s}\left(2 s C_{x}\left(\eta_{x}^{2-2 s}-\varepsilon^{2-2 s}\right) A_{\varepsilon}+\left(\frac{\eta_{x}^{-2 s}}{2}+\frac{s \eta_{x}^{1-2 s}}{2 s-1}\right) \cdot(1-s) \omega_{\phi}\left(A_{\varepsilon}\right)\right) \\
& +2 s \varepsilon^{3} \frac{\left|\nabla^{2} \phi(x)\right|^{2}}{\left|p_{x}\right|}+s \varepsilon^{2} \sup _{y \in B_{\varepsilon}(x)}\left|\nabla^{2} \phi(y)-\nabla^{2} \phi(x)\right| .
\end{aligned}
$$

For a discussion of the error terms in the above results, we refer to Remarks 3.3 and 4.2 , In particular, for $\phi$ Lipschitz, the bound in Theorem 1.1 becomes: $O\left(\varepsilon^{4 s-1}+\varepsilon^{2}\right)$ with constants that blow up as $s \rightarrow 1-$. On the other hand, the bound in Theorem 1.2 has the form: $O\left(\varepsilon^{4 s-1}\right)+o\left(\varepsilon^{2}\right)$ however the related constants are uniform in $s \in\left(\frac{1}{2}, 1\right)$, and it also is compatible with the expected error bound for the (local) $\infty$-Laplacian. This improvement is obtained by correcting the singular part of $\Delta_{\infty}^{s}$ by the corresponding asymptotic expansion of its local counterpart. Such idea was already present in the numerical analysis literature, where it was used to obtain higher order monotone numerical schemes for the fractional Laplacian and other linear nonlocal operators, see e.g. [7, 8]. The fact that the singular part of a nonlinear and nonlocal operator encodes a local counterpart is an idea also present in [4, 9].

Other asymptotic expansions for nonlocal operators such as $\Delta_{\infty}^{s}$ have been recently introduced in [3,4]. The related average in [4, section 3.2] distinguishes between the cases $p_{x} \neq 0$ 
and $p_{x}=0$. In comparison, $\mathcal{A}_{\varepsilon}$ in the present paper neither relies on this distinction nor even necessitates the notion of the gradient being well posed. Thus, they can be applied on a larger class of functions $\phi$ that are only bounded Borel.

In section [5.1, we further propose a version $\overline{\mathcal{A}}_{\varepsilon}^{o}$ of the average $\mathcal{A}_{\varepsilon}^{o}$ and its corresponding expansion, in which integration takes place on an open, bounded domain in $\mathbb{R}^{N}$, rather than an infinite line. We believe that this correction will be of importance in the implementation of numerical schemes. We also conjecture that the expected values of the stochastic process whose dynamic programming principle is modeled on $\overline{\mathcal{A}}_{\varepsilon}^{o}$ converge to these solutions in the limit $\varepsilon \rightarrow 0+$, as in the pivotal study [13] of the classical operator $\Delta_{\infty}$.

Outline of the paper. We prove (1.8) in section 2, Theorem 1.1 in section 3, and Theorem 1.2 in section 4. In section 5, we discuss $\overline{\mathcal{A}}_{\varepsilon}^{o}$ and put Theorems 1.1, 1.2 in a viscosity solution framework.

\section{The fractional $\infty$-Laplacian and a proof of (1.8)}

Given a bounded Borel function $\phi: \mathbb{R}^{N} \rightarrow \mathbb{R}$, and two parameters $\varepsilon>0$ and $s \in\left(\frac{1}{2}, 1\right)$, we will be concerned with values of the integral operators $\mathcal{L}_{s}^{\varepsilon}[\phi]: \mathbb{R}^{N} \rightarrow \mathbb{R}$, given in:

$$
\begin{aligned}
\mathcal{L}_{s}^{\varepsilon}[\phi](x) & \doteq \sup _{|y|=1} \inf _{|\tilde{y}|=1} \int_{\varepsilon}^{\infty} L_{\phi}(x, t y, t \tilde{y}) \mathrm{d} \mu_{s}(t) \\
& =\sup _{|y|=1} \int_{\varepsilon}^{\infty} \phi(x+t y) \mathrm{d} \mu_{s}(t)+\inf _{|y|=1} \int_{\varepsilon}^{\infty} \phi(x+t y) \mathrm{d} \mu_{s}(t)-\frac{(1-s) c_{s}}{\varepsilon^{2 s}} \phi(x),
\end{aligned}
$$

Note that, since the restriction of $\phi$ to any one-dimensional line is also Borel, the function $(0, \infty) \ni t \mapsto L_{\phi}(x, t y, t \tilde{y})$ is bounded and Borel for any $x, y, \tilde{y}$. Further, since $\mu_{s}(\varepsilon, \infty)=$ $\frac{\alpha_{s}}{2 s \varepsilon^{2 s}}<\infty$, each integral $\int_{\varepsilon}^{\infty} L_{\phi}(x, t y, t \tilde{y}) \mathrm{d} \mu_{s}(t)$ and consequently also the quantities $\mathcal{L}_{s}^{\varepsilon}[\phi](x)$, are all well defined and finite. On the other hand, $\mu_{s}(0, \infty)=\infty$, so neither the definition of $\mathcal{L}_{s}[\phi]$ in (1.9) nor a version of its equivalent formulation as in $\mathcal{L}_{s}^{\varepsilon}[\phi]$ are necessarily valid, when $\phi$ is only bounded and Borel. However, one immediate consequence of (1.7) is that:

$$
\left|L_{\phi}(x, t y, t \tilde{y})-t\left\langle p_{x}, y-\tilde{y}\right\rangle\right| \leq 2 C_{x} t^{2} \quad \text { for all }|y|,|\tilde{y}|=1 \text { and }|t|<\eta_{x}
$$

which yields (through an application of Taylor's expansion):

Lemma 2.1. Let $\phi \in C^{1,1}(x)$ be a bounded Borel function. Then $\left\{\mathcal{L}_{s}^{\varepsilon}[\phi](x)\right\}_{\varepsilon>0}$ are bounded independently of $\varepsilon$ and $\mathcal{L}_{s}[\phi](x)$ is well defined. More precisely, for all $\varepsilon<\eta_{x}$ there holds:

$$
\left|\mathcal{L}_{s}^{\varepsilon}[\phi](x)\right|,\left|\mathcal{L}_{s}[\phi](x)\right| \leq 2 c_{s}(1-s)\|\phi\|_{L^{\infty}} \eta_{x}^{-2 s}+c_{s} s \cdot C_{x} \eta_{x}^{2-2 s} .
$$

Proof. We use (2.2) to obtain, for any $|y|=|\tilde{y}|=1$ :

$$
\begin{gathered}
\left|\int_{\varepsilon}^{\infty} L_{\phi}(x, t y, t \tilde{y}) \mathrm{d} \mu_{s}(t)-\int_{\varepsilon}^{\eta_{x}} t\left\langle p_{x}, y-\tilde{y}\right\rangle \mathrm{d} \mu_{s}(t)\right| \leq \int_{\eta_{x}}^{\infty} 4\|\phi\|_{L^{\infty}} \mathrm{d} \mu_{s}(t)+\int_{\varepsilon}^{\eta_{x}} 2 C_{x} t^{2} \mathrm{~d} \mu_{s}(t) \\
=2 \frac{\alpha_{s}}{s \eta_{x}^{2 s}}\|\phi\|_{L^{\infty}}+\alpha_{s} C_{x} \frac{\eta_{x}^{2-2 s}-\varepsilon^{2-2 s}}{1-s} .
\end{gathered}
$$

On the other hand:

$$
\sup _{|y|=1} \inf _{|\tilde{y}|=1} \int_{\varepsilon}^{\eta_{x}} t\left\langle p_{x}, y-\tilde{y}\right\rangle \mathrm{d} \mu_{s}(t)=\int_{\varepsilon}^{\eta_{x}} t \mathrm{~d} \mu_{s}(t) \cdot \sup _{|y|=1} \inf _{|\tilde{y}|=1}\left\langle p_{x}, y-\tilde{y}\right\rangle=0 .
$$


This results in:

$$
\begin{aligned}
\left|\mathcal{L}_{s}^{\varepsilon}[\phi](x)\right| & =\left|\mathcal{L}_{s}^{\varepsilon}[\phi](x)-\sup _{|y|=1} \inf _{|\tilde{y}|=1} \int_{\varepsilon}^{\eta_{x}} t\left\langle p_{x}, y-\tilde{y}\right\rangle \mathrm{d} \mu_{s}(t)\right| \\
& \leq \sup _{|y|=|\tilde{y}|=1}\left|\int_{\varepsilon}^{\infty} L_{\phi}(x, t y, t \tilde{y}) \mathrm{d} \mu_{s}(t)-\int_{\varepsilon}^{\eta_{x}} t\left\langle p_{x}, y-\tilde{y}\right\rangle \mathrm{d} \mu_{s}(t)\right|,
\end{aligned}
$$

which proves the bound for $\left|\mathcal{L}_{s}^{\varepsilon}[\phi](x)\right|$. The bound for $\left|\mathcal{L}_{s}[\phi](x)\right|$ follows similarly.

Our proofs throughout the paper largely depend on analyzing the behaviour of approximate extremizers $y, \tilde{y}$ in the definition (2.1) We now observe that for the operator $\mathcal{L}_{s}[\phi]$ these extremizers are explicit, for a generic function $\phi$.

Proposition 2.2. Let $\phi \in C^{1,1}(x)$ be a bounded Borel function such that $p_{x} \neq 0$. Then:

$$
\mathcal{L}_{s}[\phi](x)=\int_{0}^{\infty} L_{\phi}\left(x, t \frac{p_{x}}{\left|p_{x}\right|}, t \frac{p_{x}}{\left|p_{x}\right|}\right) \mathrm{d} \mu_{s}(t)
$$

Proof. For $\delta>0$, let $y_{\delta}$ be such that $\mathcal{L}_{s}[\phi](x) \leq \inf _{|\tilde{y}|=1} \int_{0}^{\infty} L_{\phi}\left(x, t y_{\delta}, t \tilde{y}\right) \mathrm{d} \mu_{s}(t)+\delta$. Splitting the integral and applying (2.2) on the interval $\left(0, \eta_{x}\right)$ yields:

$$
\mathcal{L}_{s}[\phi](x) \leq \int_{0}^{\infty} L_{\phi}\left(x, t y_{\delta}, t \frac{p_{x}}{\left|p_{x}\right|}\right) \mathrm{d} \mu_{s}(t)+\delta \leq \int_{0}^{\eta_{x}} t\left\langle p_{x}, y_{\delta}-\frac{p_{x}}{\left|p_{x}\right|}\right\rangle \mathrm{d} \mu_{s}(t)+C+\delta,
$$

where the constant $C$ depends on $s$ and $\phi$. Using the lower bound in (2.3), we conclude that:

$$
\left\langle p_{x}, \frac{p_{x}}{\left|p_{x}\right|}-y_{\delta}\right\rangle \cdot \int_{0}^{\eta_{x}} t \mathrm{~d} \mu_{s}(t)<\infty
$$

Since the product above is nonnegative while the integral diverges to $\infty$, we get $y_{\delta}=\frac{p_{x}}{\left|p_{x}\right|}$ and:

$$
\mathcal{L}_{s}[\phi](x)=\inf _{|\tilde{y}|=1} \int_{0}^{\infty} L_{\phi}\left(x, t \frac{p_{x}}{\left|p_{x}\right|}, t \tilde{y}\right) \mathrm{d} \mu_{s}(t) .
$$

Let now $\tilde{y}_{\delta}$ be such that $\mathcal{L}_{s}[\phi](x) \geq \int_{0}^{\infty} L_{\phi}\left(x, t \frac{p_{x}}{p_{x}}, t \tilde{y}_{\delta}\right) \mathrm{d} \mu_{s}(t)-\delta$. As before, in virtue of (2.2) we get: $\mathcal{L}_{s}[\phi](x) \geq \int_{0}^{\eta_{x}} t\left\langle p_{x}, \frac{p_{x}}{\left|p_{x}\right|}-\tilde{y}_{\delta}\right\rangle \mathrm{d} \mu_{s}(t)-C-\delta$, so the upper bound in (2.3) gives:

$$
\left\langle p_{x}, \frac{p_{x}}{\left|p_{x}\right|}-\tilde{y}_{\delta}\right\rangle \cdot \int_{0}^{\eta_{x}} t \mathrm{~d} \mu_{s}(t)<\infty
$$

Consequently $\tilde{y}_{\delta}=\frac{p_{x}}{\left|p_{x}\right|}$, so that: $\mathcal{L}_{s}[\phi](x) \geq \int_{0}^{\infty} L_{\phi}\left(x, t \frac{p_{x}}{\left|p_{x}\right|}, t \frac{p_{x}}{\left|p_{x}\right|}\right) \mathrm{d} \mu_{s}(t)-\delta$ for all $\delta>0$. The proof is done, in view of (2.5).

Note that formulation (2.4) corresponds, up to a constant, to the one given in [1, Definition 1.1] for $p_{x} \neq 0$. The case $p_{x}=0$ in that definition is equivalent to (1.8).

\section{A proof of Theorem 1.1}

We first observe that taking $p_{x}=0$ in (2.2) implies the following bound, for all $\varepsilon<\eta_{x}$ :

$$
\begin{aligned}
\left|\mathcal{L}_{s}^{\varepsilon}[\phi](x)-\mathcal{L}_{s}[\phi](x)\right| & \leq \sup _{|y|=|\tilde{y}|=1}\left|\int_{\varepsilon}^{\infty} L_{\phi}(x, t y, t \tilde{y}) \mathrm{d} \mu_{s}(t)-\int_{0}^{\infty} L_{\phi}(x, t y, t \tilde{y}) \mathrm{d} \mu_{s}(t)\right| \\
& \leq \int_{0}^{\varepsilon} 2 C_{x} t^{2} \mathrm{~d} \mu_{s}(t)=c_{s} s \cdot C_{x} \varepsilon^{2-2 s} .
\end{aligned}
$$


In order to estimate the same difference when $p_{x} \neq 0$, we will quantify estimates in the proof of Proposition 2.2 for higher regular functions, as specified below.

Proposition 3.1. Assume $(\underline{\mathbf{H}})$ with $p_{x} \neq 0$. Then, for every $\varepsilon<\eta_{x}$ there holds:

$$
\begin{aligned}
\mid \mathcal{L}_{s}^{\varepsilon}[\phi](x)- & \int_{\varepsilon}^{\infty} L_{\phi}\left(x, t \frac{p_{x}}{\left|p_{x}\right|}, t \frac{p_{x}}{\left|p_{x}\right|}\right) \mathrm{d} \mu_{s}(t) \mid \\
& \leq 4 c_{s} s \cdot C_{x}\left(\eta_{x}^{2-2 s}-\varepsilon^{2-2 s}\right) \cdot A_{\varepsilon}+c_{s}(1-s) \cdot\left(\eta_{x}^{-2 s}+\frac{2 s}{2 s-1} \eta_{x}^{1-2 s}\right) \cdot \omega_{\phi}\left(A_{\varepsilon}\right),
\end{aligned}
$$

Proof. 1. For every $\varepsilon<\eta_{x}$ and every small $\delta>0$ let $\left|y_{\delta}^{\varepsilon}\right|=1$ satisfy: $\sup _{|y|=1} \int_{\varepsilon}^{\infty} \phi(x+$ ty) $\mathrm{d} \mu_{s}(t) \leq \int_{\varepsilon}^{\infty} \phi\left(x+t y_{\delta}^{\varepsilon}\right) \mathrm{d} \mu_{s}(t)+\delta$. In particular, this implies:

$$
\int_{\varepsilon}^{\infty} \phi\left(x+t \frac{p_{x}}{\left|p_{x}\right|}\right) \mathrm{d} \mu_{s}(t) \leq \int_{\varepsilon}^{\infty} \phi\left(x+t y_{\delta}^{\varepsilon}\right) \mathrm{d} \mu_{s}(t)+\delta
$$

Denote $A=\left|\frac{p_{x}}{\left|p_{x}\right|}-y_{\delta}^{\varepsilon}\right|$. Together with (1.7), the above bound results in:

$$
\begin{aligned}
\delta & \geq \int_{\varepsilon}^{\eta_{x}}\left(\phi\left(x+t \frac{p_{x}}{\left|p_{x}\right|}\right)-\phi\left(x+t y_{\delta}^{\varepsilon}\right)\right) \mathrm{d} \mu_{s}(t)-\int_{\eta_{x}}^{\infty}\left|\phi\left(x+t \frac{p_{x}}{\left|p_{x}\right|}\right)-\phi\left(x+t y_{\delta}^{\varepsilon}\right)\right| \mathrm{d} \mu_{s}(t) \\
& \geq \int_{\varepsilon}^{\eta_{x}} t\left\langle\nabla \phi\left(x+t \frac{p_{x}}{\left|p_{x}\right|}\right), \frac{p_{x}}{\left|p_{x}\right|}-y_{\delta}^{\varepsilon}\right\rangle \mathrm{d} \mu_{s}(t)-\int_{\varepsilon}^{\eta_{x}} C_{x} t^{2} A^{2} \mathrm{~d} \mu_{s}(t)-\int_{\eta_{x}}^{\infty}(1+t) \cdot \omega_{\phi}(A) \mathrm{d} \mu_{s}(t) \\
& \geq\left\langle p_{x}, \frac{p_{x}}{\left|p_{x}\right|}-y_{\delta}^{\varepsilon}\right\rangle \int_{\varepsilon}^{\eta_{x}} t \mathrm{~d} \mu_{s}(t)-4 C_{x} A \int_{\varepsilon}^{\eta_{x}} t^{2} \mathrm{~d} \mu_{s}(t)-\omega_{\phi}(A) \int_{\eta_{x}}^{\infty}(1+t) \mathrm{d} \mu_{s}(t) .
\end{aligned}
$$

The last bound above follows by observing that $\left|\nabla \phi\left(x+t \frac{p_{x}}{\left|p_{x}\right|}\right)-\nabla \phi(x)\right| \leq 2 C_{x} t$ for all $|t| \leq \eta_{x}$ and that $A \leq 2$. Consequently, we get:

$$
\left\langle p_{x}, \frac{p_{x}}{\left|p_{x}\right|}-y_{\delta}^{\varepsilon}\right\rangle \leq \frac{1}{\int_{\varepsilon}^{\eta_{x}} t \mathrm{~d} \mu_{s}(t)}\left(\delta+4 C_{x} A \int_{\varepsilon}^{\eta_{x}} t^{2} \mathrm{~d} \mu_{s}(t)+\omega_{\phi}(A) \int_{\eta_{x}}^{\infty}(1+t) \mathrm{d} \mu_{s}(t)\right) .
$$

On the other hand, by a straightforward calculation:

$$
A^{2}=\left|\frac{p_{x}}{\left|p_{x}\right|}-y_{\delta}^{\varepsilon}\right|^{2}=2-2\left\langle\frac{p_{x}}{\left|p_{x}\right|}, y_{\delta}^{\varepsilon}\right\rangle=\frac{2}{\left|p_{x}\right|}\left\langle p_{x}, \frac{p_{x}}{\left|p_{x}\right|}-y_{\delta}^{\varepsilon}\right\rangle
$$

the last two displayed formulas yields that:

$$
A^{2} \leq \frac{2}{\left|p_{x}\right| \int_{\varepsilon}^{\eta_{x}} t \mathrm{~d} \mu_{s}(t)}\left(\delta+4 C_{x} A \int_{\varepsilon}^{\eta_{x}} t^{2} \mathrm{~d} \mu_{s}(t)+\omega_{\phi}(A) \int_{\eta_{x}}^{\infty}(1+t) \mathrm{d} \mu_{s}(t)\right) .
$$

We now simplify (3.3) as follows. Without loss of generality, we may assume that $\delta>0$ satisfies: $\delta \cdot\left|p_{x}\right| \int_{\varepsilon}^{\eta_{x}} t \mathrm{~d} \mu_{s}(t) \leq\left(16 C_{x} \int_{\varepsilon}^{\eta_{x}} t^{2} \mathrm{~d} \mu_{s}(t)\right)^{2}$, In case when $\delta$ is larger than the two other terms in the right hand side of (3.3), we get:

$$
A^{2} \leq \frac{4 \delta}{\left|p_{x}\right| \int_{\varepsilon}^{\eta_{x}} t \mathrm{~d} \mu_{s}(t)} \leq\left(\frac{32 C_{x} \int_{\varepsilon}^{\eta_{x}} t^{2} \mathrm{~d} \mu_{s}(t)}{\left|p_{x}\right| \int_{\varepsilon}^{\eta_{x}} t \mathrm{~d} \mu_{s}(t)}\right)^{2},
$$

In the opposite case, there holds:

$$
A^{2} \leq \frac{4}{\left|p_{x}\right| \int_{\varepsilon}^{\eta_{x}} t \mathrm{~d} \mu_{s}(t)}\left(4 C_{x} A \int_{\varepsilon}^{\eta_{x}} t^{2} \mathrm{~d} \mu_{s}(t)+\omega_{\phi}(A) \int_{\eta_{x}}^{\infty}(1+t) \mathrm{d} \mu_{s}(t)\right) \doteq I_{1}+I_{2} .
$$


Further, when $I_{2} \leq I_{1}$, then we obtain the same bound as in (3.4), namely:

$$
A \leq \frac{32 C_{x} \int_{\varepsilon}^{\eta_{x}} t^{2} \mathrm{~d} \mu_{s}(t)}{\left|p_{x}\right| \int_{\varepsilon}^{\eta_{x}} t \mathrm{~d} \mu_{s}(t)}=\frac{16 C_{x}}{\left|p_{x}\right|} \cdot \frac{2 s-1}{1-s} \cdot \frac{\eta_{x}^{2-2 s}-\varepsilon^{2-2 s}}{\varepsilon^{1-2 s}-\eta_{x}^{1-2 s}} .
$$

On the other hand, $I_{1}<I_{2}$ implies:

$$
A^{2} \leq \frac{8 \omega_{\phi}(A) \int_{\eta_{x}}^{\infty}(1+t) \mathrm{d} \mu_{s}(t)}{\left|p_{x}\right| \int_{\varepsilon}^{\eta_{x}} t \mathrm{~d} \mu_{s}(t)}=\frac{8 \omega_{\phi}(A)}{\left|p_{x}\right|} \cdot \frac{\frac{2 s-1}{2 s} \eta_{x}^{-2 s}+\eta_{x}^{1-2 s}}{\varepsilon^{1-2 s}-\eta_{x}^{1-2 s}} .
$$

We hence conclude that $A \leq A_{\varepsilon}$ in either of the above cases.

2. Similarly as in step 1 , we see that the unit vector $\tilde{y}_{\delta}^{\varepsilon}$ with the property: $\inf _{|y|=1} \int_{\varepsilon}^{\infty} \phi(x-$ ty) $\mathrm{d} \mu_{s}(t) \geq \int_{\varepsilon}^{\infty} \phi\left(x-t \tilde{y}_{\delta}^{\varepsilon}\right) \mathrm{d} \mu_{s}(t)-\delta$, satisfies: $\left|\frac{p_{x}}{\left|p_{x}\right|}-\tilde{y}_{\delta}^{\varepsilon}\right| \leq A_{\varepsilon}$. We now write:

$$
\int_{\varepsilon}^{\infty} L_{\phi}\left(x, t \tilde{y}_{\delta}^{\varepsilon}, t \tilde{y}_{\delta}^{\varepsilon}\right) \mathrm{d} \mu_{s}(t)-\delta \leq \mathcal{L}_{s}^{\varepsilon}[\phi](x) \leq \int_{\varepsilon}^{\infty} L_{\phi}\left(x, t y_{\delta}^{\varepsilon}, t y_{\delta}^{\varepsilon}\right) \mathrm{d} \mu_{s}(t)+\delta,
$$

which implies:

$$
\begin{aligned}
& \left|\mathcal{L}_{s}^{\varepsilon}[\phi](x)-\int_{\varepsilon}^{\infty} L_{\phi}\left(x, t \frac{p_{x}}{\left|p_{x}\right|}, t \frac{p_{x}}{\left|p_{x}\right|}\right) \mathrm{d} \mu_{s}(t)\right| \leq \delta+\max \left\{\left|I\left(y_{\delta}^{\varepsilon}\right)\right|,\left|I\left(\tilde{y}_{\delta}^{\varepsilon}\right)\right|\right\}, \\
& \text { where: } I(y) \doteq \int_{\varepsilon}^{\infty} \phi(x+t y)-\phi\left(x+t \frac{p_{x}}{\left|p_{x}\right|}\right)+\phi(x-t y)-\phi\left(x-t \frac{p_{x}}{\left|p_{x}\right|}\right) \mathrm{d} \mu_{s}(t) .
\end{aligned}
$$

Observe that:

$$
\begin{aligned}
\left|I\left(y_{\delta}^{\varepsilon}\right)\right| \leq & \left|\int_{\varepsilon}^{\eta_{x}} \phi\left(x+t y_{\delta}^{\varepsilon}\right)-\phi\left(x+t \frac{p_{x}}{\left|p_{x}\right|}\right)+\phi\left(x-t y_{\delta}^{\varepsilon}\right)-\phi\left(x-t \frac{p_{x}}{\left|p_{x}\right|}\right) \mathrm{d} \mu_{s}(t)\right| \\
& +\int_{\eta_{x}}^{\infty}\left|\phi\left(x+t y_{\delta}^{\varepsilon}\right)-\phi\left(x+t \frac{p_{x}}{\left|p_{x}\right|}\right)\right|+\left|\phi\left(x-t y_{\delta}^{\varepsilon}\right)-\phi\left(x-t \frac{p_{x}}{\left|p_{x}\right|}\right)\right| \mathrm{d} \mu_{s}(t) \doteq \bar{I}_{1}+\bar{I}_{2} .
\end{aligned}
$$

In order to deal with $\bar{I}_{1}$, we use the Taylor expansion:

$$
\left|\phi\left(x \pm t y_{\delta}^{\varepsilon}\right)-\phi\left(x \pm t \frac{p_{x}}{\left|p_{x}\right|}\right)-\left\langle\nabla \phi\left(x \pm t \frac{p_{x}}{\left|p_{x}\right|}\right), \pm t\left(y_{\delta}^{\varepsilon}-\frac{p_{x}}{\left|p_{x}\right|}\right)\right\rangle\right| \leq C_{x} t^{2}\left|y_{\delta}^{\varepsilon}-\frac{p_{x}}{\left|p_{x}\right|}\right|^{2}
$$

which upon integration implies:

$$
\bar{I}_{1} \leq \int_{\varepsilon}^{\eta_{x}} C_{x} t^{2}\left(4 A+2 A^{2}\right) \mathrm{d} \mu_{s}(t) \leq 8 C_{x} A \int_{\varepsilon}^{\eta_{x}} t^{2} \mathrm{~d} \mu_{s}(t)=4 C_{x} \alpha_{s} \frac{\eta_{x}^{2-2 s}-\varepsilon^{2-2 s}}{1-s} \cdot A .
$$

For the term $\bar{I}_{2}$, we get:

$$
\bar{I}_{2} \leq 2 \int_{\eta_{x}}^{\infty}(1+t) \cdot \omega_{\phi}(A) \mathrm{d} \mu_{s}(t)=2 \alpha_{s}\left(\frac{\eta_{x}^{-2 s}}{2 s}+\frac{\eta_{x}^{1-2 s}}{2 s-1}\right) \cdot \omega_{\phi}(A) .
$$

In conclusion, we obtain the following bounds:

$$
\left|I\left(y_{\delta}^{\varepsilon}\right)\right|, \quad\left|I\left(\tilde{y}_{\delta}^{\varepsilon}\right)\right| \leq 4 C_{x} \alpha_{s} \frac{\eta_{x}^{2-2 s}-\varepsilon^{2-2 s}}{1-s} A_{\varepsilon}+2 \alpha_{s}\left(\frac{\eta_{x}^{-2 s}}{2 s}+\frac{\eta_{x}^{1-2 s}}{2 s-1}\right) \cdot \omega_{\phi}\left(A_{\varepsilon}\right) .
$$

This ends the proof in virtue of (3.5).

Corollary 3.2. Under the same assumptions and notation as in Proposition 3.1, we have:

$$
\begin{aligned}
& \left|\mathcal{L}_{s}^{\varepsilon}[\phi](x)-\mathcal{L}_{s}[\phi](x)\right| \\
& \quad \leq 4 c_{s} s \cdot C_{x}\left(\eta_{x}^{2-2 s}-\varepsilon^{2-2 s}\right) \cdot A_{\varepsilon}+c_{s}(1-s) \cdot\left(\eta_{x}^{-2 s}+\frac{2 s}{2 s-1} \eta_{x}^{1-2 s}\right) \cdot \omega_{\phi}\left(A_{\varepsilon}\right)+c_{s} s \cdot C_{x} \varepsilon^{2-2 s} .
\end{aligned}
$$


Proof. Observe that for all $t<\eta_{x}$ there holds:

$$
\left|L_{\phi}\left(x, t \frac{p_{x}}{\left|p_{x}\right|}, t \frac{p_{x}}{\left|p_{x}\right|}\right)\right| \leq t^{2}\left\|\nabla^{2} \phi\right\|_{L^{\infty}\left(B_{t}\right)} .
$$

Consequently and in view of Proposition 2.2 we get:

$$
\left|\mathcal{L}_{s}[\phi](x)-\int_{\varepsilon}^{\infty} L_{\phi}\left(x, t \frac{p_{x}}{\left|p_{x}\right|}, t \frac{p_{x}}{\left|p_{x}\right|}\right) \mathrm{d} \mu_{s}(t)\right| \leq \int_{0}^{\varepsilon}\left|L_{\phi}\left(x, t \frac{p_{x}}{\left|p_{x}\right|}, t \frac{p_{x}}{\left|p_{x}\right|}\right)\right| \mathrm{d} \mu_{s}(t) \leq \frac{\alpha_{s}}{1-s} \cdot C_{x} \varepsilon^{2-2 s} .
$$

This achieves the proof by Proposition 3.1.

Note that the bound in Corollary 3.2 is essentially valid in both cases $p_{x} \neq 0$ and $p_{x}=0$, because of (3.1). Scaling the said bound by the factor $\frac{s \varepsilon^{2 s}}{\alpha_{s}}$, we directly deduce Theorem 1.1,

Remark 3.3. (i) Observing that: $\omega_{\phi}(a) \leq 2\|\phi\|_{L^{\infty}}$, we get for all $\varepsilon<\frac{\eta_{x}}{2}$ :

$$
\kappa_{\varepsilon} \leq\left(\frac{16\|\phi\|_{L^{\infty}}}{\left|p_{x}\right|} \cdot \frac{\frac{2 s-1}{2 s} \eta_{x}^{-2 s}+\eta_{x}^{1-2 s}}{\varepsilon^{1-2 s}-\eta_{x}^{1-2 s}}\right)^{1 / 2} \leq 8\left(\frac{\|\phi\|_{L^{\infty}}}{\left|p_{x}\right|} \cdot \frac{\eta_{x}^{-2 s}+\eta_{x}^{1-2 s}}{2 s-1}\right)^{1 / 2} \varepsilon^{s-1 / 2} .
$$

In the second inequality we used that for all $\varepsilon<\frac{\eta_{x}}{2}$ and all $s \in\left(\frac{1}{2}, 1\right)$ there holds:

$$
\varepsilon^{1-2 s}-\eta_{x}^{1-2 s}>\varepsilon^{1-2 s}\left(1-2^{1-2 s}\right), \quad 1-2^{1-2 s} \geq(2 s-1) \ln \sqrt{2}>\frac{2 s-1}{4} .
$$

The first quantity in $A_{\varepsilon}$ is of order $\varepsilon^{2 s-1}$, so the right hand side in (3.2) is:

$$
C(s) \cdot C\left(C_{x}, \frac{1}{\left|p_{x}\right|},\|\phi\|_{L^{\infty}}\right) \cdot C\left(\eta_{x}\right) \varepsilon^{s-1 / 2}+C(s) \cdot C\left(\frac{1}{\left|p_{x}\right|},\|\phi\|_{L^{\infty}}\right) \cdot C\left(\eta_{x}\right) \omega_{\phi}\left(\varepsilon^{s-1 / 2}\right),
$$

where $C(s)$ depends only on $s$ and $C\left(\eta_{x}\right)$ only on $\eta_{x}$ and the remaining constants depend on the other displayed terms, in a nondecreasing manner.

(ii) When $\phi \in C^{0, \alpha}\left(\mathbb{R}^{N} \backslash \bar{B}_{\eta_{x}}\right)$ with $\alpha \in(0,1)$, then $\omega_{\phi}(a)=[\phi]_{\alpha} a^{\alpha}$. Therefore:

$$
\kappa_{\varepsilon} \leq\left(\frac{32[\phi]_{\alpha}}{\left|p_{x}\right|} \cdot \frac{\eta_{x}^{-2 s}+\eta_{x}^{1-2 s}}{2 s-1}\right)^{\frac{1}{2-\alpha}} \varepsilon^{\frac{2 s-1}{2-\alpha}}
$$

whereas (3.2) can be replaced with: $C(s) \cdot C\left(\frac{1}{\mid p_{x}},[\phi]_{\alpha}\right) \cdot C\left(\eta_{x}\right) \varepsilon^{\alpha \cdot \frac{2 s-1}{2-\alpha}}$.

(iii) Finally, for $\phi$ Lipschitz on $\mathbb{R}^{N} \backslash \bar{B}_{\eta_{x}}$ with the Lipschitz constant $\operatorname{Lip}_{\phi}$, we get:

$\kappa_{\varepsilon} \leq \frac{32 \operatorname{Lip}_{\phi}}{\left|p_{x}\right|} \cdot \frac{\eta_{x}^{-2 s}+\eta_{x}^{1-2 s}}{2 s-1} \varepsilon^{2 s-1}, \quad A_{\varepsilon} \leq \frac{32}{\left|p_{x}\right|} \cdot \max \left\{\frac{2 C_{x} \eta_{x}^{2-2 s}}{1-s}, \frac{\operatorname{Lip}_{\phi}\left(\eta_{x}^{-2 s}+\eta_{x}^{1-2 s}\right)}{2 s-1}\right\} \varepsilon^{2 s-1}$.

Indeed, both quantities in $A_{\varepsilon}$ have $\varepsilon^{2 s-1}$-order. The expression in (3.2) is then: $C(s)$. $C\left(C_{x}, \frac{1}{\left|p_{x}\right|}, \operatorname{Lip}_{\phi}\right) \cdot C\left(\eta_{x}\right) \varepsilon^{2 s-1}$, whereas the order of the error bounding quantity in Theorem 1.1 is $C(s) \cdot\left(\varepsilon^{4 s-1}+\varepsilon^{2}\right)$ as $\varepsilon \rightarrow 0+$, and $C(s) \rightarrow \infty$ as $s \rightarrow 1-$.

\section{A proof of Theorem 1.2}

We note the following refinement of the argument in the proof of Corollary 3.2 
Proposition 4.1. Let $\phi \in C^{2}\left(\bar{B}_{\eta_{x}}\right)$ satisfy: $p_{x} \doteq \nabla \phi(x) \neq 0$. Then, for every $\varepsilon<\eta_{x}$ such that $\varepsilon\left|\nabla^{2} \phi(x)\right| \leq\left|p_{x}\right|$, there holds:

$$
\begin{aligned}
\mid c_{s} s \cdot \varepsilon^{-2 s} \cdot \frac{1}{2}\left(\sup _{B_{\varepsilon}(x)} \phi\right. & \left.+\inf _{B_{\varepsilon}(x)} \phi-2 \phi(x)\right)-\int_{0}^{\varepsilon} L_{\phi}\left(x, t \frac{p_{x}}{\left|p_{x}\right|}, t \frac{p_{x}}{\left|p_{x}\right|}\right) \mathrm{d} \mu_{s}(t) \mid \\
\leq & c_{s} s\left(2 \varepsilon^{3-2 s} \frac{\left|\nabla^{2} \phi(x)\right|^{2}}{\left|p_{x}\right|}+\varepsilon^{2-2 s} \sup _{y \in B_{\varepsilon}(x)}\left|\nabla^{2} \phi(y)-\nabla^{2} \phi(x)\right|\right) .
\end{aligned}
$$

Proof. A simple application of Taylor's expansion yields:

$$
\left|L_{\phi}\left(x, t \frac{p_{x}}{\left|p_{x}\right|}, t \frac{p_{x}}{\left|p_{x}\right|}\right)-t^{2} \Delta_{\infty} \phi(x)\right| \leq t^{2} \sup _{y \in B_{t}}\left|\nabla^{2} \phi(y)-\nabla^{2} \phi(x)\right|,
$$

where we recall that $\Delta_{\infty} \phi(x)=\left\langle\nabla^{2} \phi(x): \frac{p_{x}}{\left|p_{x}\right|} \otimes \frac{p_{x}}{\left|p_{x}\right|}\right\rangle$. Integrating the above $\int_{0}^{\varepsilon} \mathrm{d} \mu_{s}(t)$, we get: $\left|\int_{0}^{\varepsilon} L_{\phi}\left(x, t \frac{p_{x}}{\left|p_{x}\right|}, t \frac{p_{x}}{\left|p_{x}\right|}\right) \mathrm{d} \mu_{s}(t)-\frac{\alpha_{s}}{2(1-s)} \varepsilon^{2-2 s} \Delta_{\infty} \phi(x)\right| \leq \frac{\alpha_{s}}{2(1-s)} \varepsilon^{2-2 s} \sup _{y \in B_{\varepsilon}}\left|\nabla^{2} \phi(y)-\nabla^{2} \phi(x)\right|$.

Recalling that (see for example [11, section 3.2]):

$$
\left|\left(\sup _{B_{\varepsilon}} \phi+\inf _{B_{\varepsilon}} \phi-2 \phi(x)\right)-\varepsilon^{2} \Delta_{\infty} \phi(x)\right| \leq 4 \varepsilon^{3} \frac{\left|\nabla^{2} \phi(x)\right|^{2}}{\left|p_{x}\right|}+\varepsilon^{2} \sup _{y \in B_{\varepsilon}}\left|\nabla^{2} \phi(y)-\nabla^{2} \phi(x)\right|,
$$

and taking the linear combination of the two above formulas, the proof is done.

The proof of Theorem 1.2 follows directly by summing up formulas (3.2), (4.1), and multiplying the result by the factor $\frac{(1-s) s}{\alpha_{s}} \varepsilon^{2 s}$. Since:

$$
\left(\frac{(1-s) s}{\alpha_{s}} \varepsilon^{2 s}\right) \mathcal{L}_{s}^{\varepsilon}[\phi](x)+\left(\frac{(1-s) s}{\alpha_{s}} \varepsilon^{2 s}\right) \cdot \frac{\alpha_{s}}{2(1-s)} \varepsilon^{-2 s}\left(\sup _{B_{\varepsilon}} \phi+\inf _{B_{\varepsilon}} \phi-2 \phi(x)\right)=\mathcal{A}_{\varepsilon} \phi(x)-\phi(x),
$$

the error in the claimed expansion is the sum of errors in (3.2) and (4.1), multiplied by $\frac{(1-s) s}{\alpha_{s}} \varepsilon^{2 s}$.

Remark 4.2. (i) Analysis similar to Remark 3.3 allows for computing the order of the error term in Theorem 1.2 when $\phi$ is Lipschitz:

$$
C(s) \cdot C\left(C_{x}, \frac{1}{\left|p_{x}\right|}, \operatorname{Lip}_{\phi}\right) \cdot C\left(\eta_{x}\right) \varepsilon^{4 s-1}+C(s) \cdot C\left(\left|\nabla^{2} \phi(x)\right|, \frac{1}{\left|p_{x}\right|}\right) \varepsilon^{3}+C(s) \cdot o\left(\varepsilon^{2}\right) .
$$

As before, $C(s)$ depends only on $s$, and $C\left(\eta_{x}\right)$ only on $\eta_{x}$, while the remaining constants depend on the displayed terms in a nondecreasing manner. For $\phi \in C^{2,1}\left(B_{\eta_{x}}\right)$, the above quantity has order $\varepsilon^{4 s-1}+\varepsilon^{3}$, which equals $\varepsilon^{3}$ at $s=1$.

(ii) For a more precise analysis of the asymptotic expansion when $s \rightarrow 1-$, note that:

$$
\begin{aligned}
& \kappa_{\varepsilon} \leq \sup \left\{a ; a \in[0,2] \text { and } a^{2} \leq \frac{32 \omega_{\phi}(a)}{\left|p_{x}\right|} \cdot \frac{\eta_{x}^{-2 s}+\eta_{x}^{1-2 s}}{2 s-1} \varepsilon^{2 s-1}\right\}, \\
& \frac{16 C_{x}}{\left|p_{x}\right|} \cdot \frac{2 s-1}{1-s} \cdot \frac{\eta_{x}^{2-2 s}-\varepsilon^{2-2 s}}{\varepsilon^{1-2 s}-\eta_{x}{ }^{1-2 s}} \leq \frac{16 C_{x}}{\left|p_{x}\right|} \cdot 16 \eta_{x}^{2-2 s}|\ln \varepsilon| \varepsilon^{2 s-1} .
\end{aligned}
$$

The first bound above is valid when $\varepsilon<\frac{\eta_{x}}{2}$, while for the second bound we used: $\eta_{x}^{2-2 s}$ $\varepsilon^{2-2 s} \leq(2-2 s)\left(\ln \eta_{x}-\ln \varepsilon\right) \eta_{x}^{2-2 s} \leq 4(1-s)|\ln \varepsilon| \eta_{x}^{2-2 s}$, when $\varepsilon<e^{-\left|\ln \eta_{x}\right|}$. Consequently, 
$A_{\varepsilon} \leq o(1)$ as $\varepsilon \rightarrow 0+$, uniformly in $s \in\left(\frac{1}{2}+\delta, 1\right)$. For each fixed $\varepsilon$, the bound in Theorem 1.2 converges to (consistently with (4.2) as $s \rightarrow 1-$ ):

$$
2 \varepsilon^{3} \frac{\left|\nabla^{2} \phi(x)\right|^{2}}{\left|p_{x}\right|}+\varepsilon^{2} \sup _{y \in B_{\varepsilon}}\left|\nabla^{2} \phi(y)-\nabla^{2} \phi(x)\right| .
$$

We also observe that when $\phi$ is Lipschitz on $\mathbb{R}^{N} \backslash \bar{B}_{\eta_{x}}$, the said bound becomes:

$$
\begin{aligned}
& (1-s) \varepsilon^{4 s-1}|\ln \varepsilon|^{2} \cdot \frac{2^{9} C_{x}}{\left|p_{x}\right|} \cdot\left(16 s C_{x} \eta_{x}^{4-4 s}+\operatorname{Lip}_{\phi} \cdot\left(\frac{\eta_{x}^{2-4 s}}{2}+\frac{s \eta_{x}^{3-4 s}}{2 s-1}\right)|\ln \varepsilon|^{-1}\right) \\
& +s\left(2 \varepsilon^{3} \frac{\left|\nabla^{2} \phi(x)\right|^{2}}{\left|p_{x}\right|}+\varepsilon^{2} \sup _{y \in B_{\varepsilon}}\left|\nabla^{2} \phi(y)-\nabla^{2} \phi(x)\right|\right) .
\end{aligned}
$$

\section{Further REMARKS}

5.1. Spherical prisms as integration domains. With an eye towards future applications, we now consider another averaging operator:

$$
\overline{\mathcal{A}}_{\varepsilon}^{o} \phi(x)=\frac{1}{2}\left(\sup _{|y|=1} f_{T^{\varepsilon, R, \alpha}(y)} \phi(x+z) \mathrm{d} \mu_{s}^{N}(z)+\inf _{|y|=1} f_{T^{\varepsilon, R, \alpha}(y)} \phi(x+z) \mathrm{d} \mu_{s}^{N}(z)\right) .
$$

Above, the integration is taken with respect to the measure $\mu_{s}^{N}$ on the Borel subsets of $\mathbb{R}^{N}$ :

$$
\mathrm{d} \mu_{s}^{N}(z) \doteq \frac{C(N, s)}{|z|^{N+2 s}} \mathrm{~d} z \quad \text { where } C(N, s)=\frac{4^{s} s \Gamma\left(\frac{N}{2}+s\right)}{\pi^{N / 2} \Gamma(1-s)}=\left(\int_{\mathbb{R}^{N}} \frac{1-\cos \left\langle z, e_{1}\right\rangle}{|z|^{N+2 s}} \mathrm{~d} z\right)^{-1}
$$

Clearly, $C(1, s)=\alpha_{s}$ and $\mu_{s}^{1}=\mu_{s}$. The integration domain $T^{\varepsilon, R, \alpha}(y)$ is the regular spherical prism in $\mathbb{R}^{N}$, oriented in the direction $y \in \mathbb{R}^{N} \backslash\{0\}$, truncated at the heights $0<\varepsilon<R$, and with the aperture angle $\angle$ determined by $\alpha>0$ as described in:

$$
T^{\varepsilon, R, \alpha}(y)=\left\{z \in \mathbb{R}^{N} ; \sin \frac{\angle(y, z)}{2}<\alpha,\langle y, z\rangle>0 \text { and } \varepsilon<|z|<R\right\} .
$$

With the above notation, $T^{0, \infty, \alpha}(y)$ is an infinite cone, and we observe that such cones were used in the definition of the fractional $p$-Laplacian $\Delta_{p}^{s}$ in [2], with $p=p(\alpha, N, s)$. We have:

Lemma 5.1. Assume (田). Then, for every $\varepsilon<\eta_{x}, R>\max \left\{\eta_{x}, 1\right\}$ and $\alpha<\frac{1}{2}$, there holds:

$$
\begin{aligned}
& \sup _{|y|=1}\left|f_{T^{\varepsilon, R, \alpha}(y)} \phi(x+z) \mathrm{d} \mu_{s}^{N}(z)-f_{\varepsilon}^{\infty} \phi(x+t y) \mathrm{d} \mu_{s}(t)\right| \\
& \leq 2\left|\frac{\varepsilon}{R}\right|^{2 s} \cdot\|\phi\|_{L^{\infty}}+\max \left\{2\left(\left|p_{x}\right|+2 C_{x} \eta_{x}\right) \eta_{x} \cdot \alpha, 3 R \cdot \omega_{\phi}(\alpha)\right\} .
\end{aligned}
$$

Proof. We first estimate the difference:

$$
\begin{aligned}
& \left|f_{\varepsilon}^{\infty} \phi(x+t y) \mathrm{d} \mu_{s}(t)-f_{\varepsilon}^{R} \phi(x+t y) \mathrm{d} \mu_{s}(t)\right| \\
& \quad \leq \frac{1}{\mu_{s}(\varepsilon, \infty)} \int_{R}^{\infty}|\phi(x+t y)| \mathrm{d} \mu_{s}(t)+\left|\frac{1}{\mu_{s}(\varepsilon, \infty)}-\frac{1}{\mu_{s}(\varepsilon, R)}\right| \int_{\varepsilon}^{R}|\phi(x+t y)| \mathrm{d} \mu_{s}(t) \\
& \quad \leq 2\left|\frac{\varepsilon}{R}\right|^{2 s} \cdot\|\phi\|_{L^{\infty}} .
\end{aligned}
$$


Next, observe that:

$$
\begin{aligned}
\int_{T^{\varepsilon, R, \alpha}(y)} \phi(x+|z| y) \mathrm{d} \mu_{s}^{N}(z) & =\int_{\varepsilon}^{R} \phi(x+t y) t^{N-1} \cdot \operatorname{area}\left(\left\{|z|=1, z \in T^{\varepsilon, R, \alpha}\right\}\right) \frac{\mathrm{d} t}{t^{N+2 s}} \\
& =\operatorname{area}\left(\left\{|z|=1, z \in T^{\varepsilon, R, \alpha}\right\}\right) \cdot \int_{\varepsilon}^{R} \phi(x+t y) \mathrm{d} \mu_{s}(t)
\end{aligned}
$$

which implies: $f_{T^{\varepsilon, R, \alpha}(y)} \phi(x+|z| y) \mathrm{d} \mu_{s}^{N}(z)=f_{\varepsilon}^{R} \phi(x+t y) \mathrm{d} \mu_{s}(t)$. It remains to bound:

$$
\begin{aligned}
f_{T^{\varepsilon, R, \alpha}(y)} & |\phi(x+z)-\phi(x+|z| y)| \mathrm{d} \mu_{s}^{N}(z) \leq \sup _{z \in T^{\varepsilon, R, \alpha}(y)}|\phi(x+z)-\phi(x+|z| y)| \\
& \leq \max \left\{\|\nabla \phi\|_{L^{\infty}\left(B_{\eta_{x}}\right)} \cdot 2 \eta_{x} \cdot \alpha, \omega_{\phi}(2 R \alpha)\right\} \\
& \leq \max \left\{2\left(\left|p_{x}\right|+2 C_{x} \eta_{x}\right) \eta_{x} \cdot \alpha,(1+2 R) \cdot \omega_{\phi}(\alpha)\right\} .
\end{aligned}
$$

This yields the desired estimate and ends the proof.

From Lemma 5.1, Remark 3.3 and Theorem 1.1, we directly deduce:

Corollary 5.2. Assume ([-

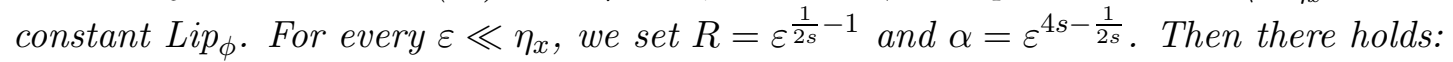

$$
\begin{aligned}
\mid \overline{\mathcal{A}}_{\varepsilon}^{o} \phi(x)-\phi(x)- & \frac{1}{c_{s}(1-s)} \varepsilon^{2 s} \mathcal{L}_{s}[\phi](x) \mid \leq \varepsilon^{4 s-1}\left(2\|\phi\|_{L^{\infty}}+3 \operatorname{Lip}_{\phi}\right)+\frac{s}{1-s} \cdot 2 C_{x} \varepsilon^{2} \\
& +\left\{\begin{array}{cc}
\frac{32}{\left|p_{x}\right|} \varepsilon^{4 s-1}\left(\frac{8 s}{1-s}+\left(\eta_{x}^{-2 s}+\frac{2 s}{2 s-1} \eta_{x}^{1-2 s}\right) \operatorname{Lip}_{\phi}\right) . \\
\cdot \max \left\{\frac{2 C_{x}}{1-s}, \frac{\left(\eta_{x}^{-2 s}+\eta_{x}^{1-2 s}\right)}{2 s-1} \text { Lip }_{\phi}\right\} & \text { when } p_{x} \neq 0 \\
0 & \text { when } p_{x}=0 .
\end{array}\right.
\end{aligned}
$$

Remark 5.3. Towards the applications in the numerical approximating of solutions to the nonlocal Dirichlet problem for the operator $\Delta_{\infty}^{s}$, one has to consider a discrete version of the result in Theorem 1.1. To this end, let $\left\{\theta_{i}\right\}_{i=1}^{n}$ be an equidistributed spherical grid on $\{|z|=1\} \subset \mathbb{R}^{N}$; when $N=2$ then $\theta_{i}=e^{2 \pi i / n}$. Next, for all $x_{k}$ in the cubical grid $h \mathbb{Z}^{N}$ define:

$$
\overline{\mathcal{A}}_{\varepsilon}^{d} \phi\left(x_{k}\right) \doteq \frac{s h^{N}}{\left|S_{\alpha}\right|\left(\varepsilon^{-2 s}-R^{-2 s}\right)} \cdot\left(\max _{i=1 \ldots n}+\min _{i=1 \ldots n}\right) \sum_{x_{j} \in T^{\varepsilon, R, \alpha}\left(\theta_{i}\right) \cap h \mathbb{Z}^{N}} \frac{\phi\left(x_{k}+x_{j}\right)}{\left|x_{j}\right|^{N+2 s}},
$$

where we used that $\mu_{s}^{N}\left(T^{\varepsilon, R, \alpha}\right)=C(N, s)\left|S_{\alpha}\right| \cdot \frac{\varepsilon^{-2 s}-R^{-2 s}}{2 s}$, with $S_{\alpha} \doteq T^{0, \infty, \alpha} \cap\{|z|=1\}$.

It is clear that for $h$ and $n$ scaling in $\varepsilon$ with sufficiently high positive and negative powers, respectively, the averaging operator $\overline{\mathcal{A}}_{\varepsilon}^{d}$ is a discrete approximation of $\mathcal{A}_{\varepsilon}^{o}$ at the same rate of the error proved in Corollary 5.2. The details of this construction as well as its implementation for a numerical scheme, are left for the future work.

5.2. The viscosity framework. We observe that our results may be reformulated in the viscosity setting, which has been used in the results of [12] for the (local) $\infty$ - Laplacian. The definition of viscosity solutions for the fractional $\infty$-Laplacian as in [1, Definition 2.3] encodes the hypothesis $(\underline{\mathbf{H}})$ which needs to be satisfied by the test functions $\phi$. Following this lead, one can consider the asymptotic expansions in the viscosity sense. From now on, the respective 
averages in (1.4), (1.5) and (5.1), are generically denoted by Average $_{\varepsilon}$, with corresponding constants $K>0$ such that:

$$
\frac{K}{\varepsilon^{2 s}}\left(\text { Average }_{\varepsilon} \phi-\phi\right)=\Delta_{\infty}^{s} \phi+o(1) \quad \text { as } \varepsilon \rightarrow 0+.
$$

Definition 5.4. Let $\Omega \subset \mathbb{R}^{N}$ be open and let $f: \Omega \rightarrow \mathbb{R}$. A bounded upper (resp. lower) semicontinuous function $u: \mathbb{R}^{N} \rightarrow \mathbb{R}$ is a viscosity sub-solution (resp. super-solution) of:

$$
\frac{K}{\varepsilon^{2 s}}\left(\text { Average }_{\varepsilon} u-u\right)=f+o(1) \quad \text { in } \Omega \quad \text { as } \varepsilon \rightarrow 0+,
$$

provided that the following holds. For every $x \in \Omega, r>0$, and $\psi \in C^{2}\left(\bar{B}_{r}(x)\right)$ such that:

$$
\psi(x)=u(x) \quad \text { and } \quad \psi(y)>u(y) \quad(\text { resp. } \psi(y)<u(y)) \quad \text { for all } y \in \bar{B}_{r}(x) \backslash\{x\},
$$

we have:

$$
\frac{K}{\varepsilon^{2 s}}\left(\text { Average }_{\varepsilon} \phi(x)-\phi(x)\right) \geq f(x)+o(1) \quad\left(\text { resp. } \frac{K}{\varepsilon^{2 s}}\left(\text { Average }_{\varepsilon} \phi(x)-\phi(x)\right) \leq f(x)+o(1)\right),
$$

where $\phi \doteq \mathbb{1}_{\bar{B}_{r}(x)} \psi+\mathbb{1}_{\mathbb{R}^{N} \backslash \bar{B}_{r}(x)} u$. When $u$ is both a viscosity sub- and super-solution, it is a viscosity solution of (5.2) (i.e. it satisfies the asymptotic expansion in the viscosity sense).

The following follows from either of Theorems 1.1, 1.2 or Corollary [5.2 in a standard fashion:

Theorem 5.5. Let $\Omega \subset \mathbb{R}^{N}$ be open, $u: \mathbb{R}^{N} \rightarrow \mathbb{R}$ be bounded and uniformly continuous, and $f: \Omega \rightarrow \mathbb{R}$. Then the following are equivalent:

(i) $u$ is a viscosity solution of: $\Delta_{\infty}^{s} u=f$ in $\Omega$,

(ii) $u$ satisfies: $\frac{K}{\varepsilon^{2 s}}\left(\right.$ Average $\left._{\varepsilon} u-u\right)=f+o(1)$ in $\Omega$ as $\varepsilon \rightarrow 0+$, in the viscosity sense.

We refer the reader to [4] for similar statements in the context of other averages for $\Delta_{\infty}^{s}$.

\section{ACKNOWLEDGEMENTS}

This research was started when M. Lewicka visited Norwegian University of Science and Technology. Part of this research was then carried out while F. del Teso and J. Endal visited the University of Pittsburgh. We want to thank both institutions for their hospitality.

\section{REFERENCES}

[1] Bjorland, C., Caffarelli, L. and Figaldi, A., Nonlocal Tug-of-War and the inifnity fractional Laplacian, Comm. Pure Appl. Math., 65, pp. 337-380, (2012).

[2] Bjorland, C., Caffarelli, L. And Figalli, A., Non-local gradient dependent operators, Adv. Math., 230(4-6), pp. 1859-1894, (2012).

[3] Bucur, C. And Squassina, M., Asymptotic mean value properties for fractional anisotropic operators, J. Math. Anal. Appl., 466(1), pp. 107-126, (2018).

[4] Bucur, C. And Squassina, M., An asymptotic expansion for the fractional p-Laplacian and for gradient dependent nonlocal operators, To appear in Communications in Contemporary Mathematics.

[5] Chambolle, A., Lindgren, E. and Monneau, R., A Hölder infinity Laplacian, ESAim Control Optim. Calc. Var., 18(3), pp. 799-835, (2012).

[6] Chasseigne, E. And Jakobsen, E.R., On nonlocal quasilinear equations and their local limits, J. Differential Equations, 262(6), pp. 3759-3804, (2017).

[7] Del Teso, F., Endal, J. And Jakobsen, E.R., Robust numerical methods for nonlocal (and local) equations of porous medium type. Part II: Schemes and experiments, SIAM J. Numer. Anal., 56(6), pp. 3611-3647, (2018).

[8] Del Teso, F. And Endal, J. and Jakobsen, E.R., Robust numerical methods for nonlocal (and local) equations of porous medium type. Part I: Theory, SIAM J. Numer. Anal., 57(5), pp. 2266-2299, (2019). 
[9] del Teso, F. And Lindgren, E., A mean value formula for the variational p-Laplacian, preprint.

[10] Di Nezza, E., Palatucci, G. and Valdinoci, E., Hitchhiker's guide to the fractional Sobolev spaces, Bull. Sci. Math., 136(5), pp. 521-573, (2012).

[11] Lewicka, M., A course on Tug-of-War games with random noise, Springer Universitext, 254 pp., (2020).

[12] Manfredi, J. J., Parviainen, M. and Rossi, J. D., An asymptotic mean value characterization for p-harmonic functions, Proc. Amer. Math. Soc., 138(3), pp. 881-889, (2010).

[13] Peres, Y., Schramm O., Sheffield, S. and Wilson, D., Tug-of-War and the infinity Laplacian, J. Amer. Math. Soc., 22, pp. 167-210, (2009).

F. del Teso: Universidad Complutense de Madrid, Departamento de Análisis Matemático y Matemática Aplicada, 28040 Madrid, Spain

J. Endal: Universidad Autónoma de Madrid, Departamento de Matemáticas, 28049 Madrid, SPAIN

M. Lewicka: University of Pittsburgh, Department of Mathematics, 139 University Place, Pittsburgh, PA 15260, USA

Email address: fdelteso@ucm.es, jorgen.endal@uam.es, lewicka@pitt.edu 